\title{
DETERMINACIÓN DE MORINA EN TÉ VERDE Y CAFÉ POR VOLTAMPEROMETRÍA DE ADSORCIÓN UTILIZANDO ELECTRODOS DE CARBONO MODIFICADOS CON POLI (3,4-ETILENDIOXITIOFENO) Y LÍQUIDO IÓNICO
}

\author{
Daniel Peña ${ }^{\mathrm{a}}$, Elkin Pulido ${ }^{\mathrm{a}}$, Manuel Otiniano ${ }^{\mathrm{b},{ }^{*}}$ Edgar Nagles $^{\mathrm{b}, \mathrm{c}}$
}

\begin{abstract}
RESUMEN
Este artículo presenta un método sensible y selectivo para la determinación de morina (MR) en presencia de rutina (RT) y quercetina (QC) en un electrodo de carbono serigrafiado (SPCE) recubierto con poli (3,4-etilendioxitiofeno) (PEDOT) y líquidos iónicos 1-butil-3tetrafluoroborato de metilimidazolio (BMIMBF4), (PEDOT-BMIMBF4 / SPCE). Varios estudios han demostrado que la morina puede adsorberse en la superficie del electrodo SPC modificado con PEDOT. Además, (BMIMBF4) en la superficie del electrodo modificado con PEDOT aumentó la corriente pico anódica casi 52,0 \% y facilitó la oxidación de la morina a valores potenciales menos positivos, demostrando ser una superficie ideal hacia la oxidación de la morina. Por otro lado, las sustancias potenciales que pueden interferir con la señal de morina, como la rutina y la quercetina, se oxidan a un potencial más y menos positivo, respectivamente. La voltamperometría cíclica $(\mathrm{CV})$ y la espectroscopía de impedancia electroquímica (EIS) se utilizaron para cuantificar y caracterizar el electrodo modificado. El pH y los parámetros electroquímicos, como el potencial de adsorción, el tiempo de adsorción y la velocidad de barrido se obtuvieron de forma óptima $\left(\mathrm{pH} 2,7 ; \mathrm{E}_{\mathrm{ADS}},-0,10 \mathrm{~V}\right.$; tADS, 60 $\left.\mathrm{s} ; \mathrm{y} v, 50 \mathrm{mVs}^{-1}\right)$. El límite de detección fue de $0,16 \mu \mathrm{mol} \mathrm{L} \mathrm{L}^{-1} \mathrm{y}$ el RSD fue del 5,2\%. La utilidad del electrodo modificado se usó para cuantificar muestras de té verde de morinina en presencia de otras flavonas con resultados consistentes.
\end{abstract}

Palabras clave: morina, té verde, voltamperometría de adsorción, líquido iónico, electrodo serigrafiado de carbono.

\footnotetext{
a Facultad de Ciencias Básicas, Universidad de la Amazonia, Calle 17 Diagonal 17, Florencia, Colombia

b Departamento de Química Analítica, Facultad de Química e Ingeniería Química, Universidad Nacional Mayor de San Marcos, Calle Germán Amézaga N 375, Lima, Perú. enaglesv@unmsm.edu.pe

c Facultad de Ciencias Naturales y Matemáticas, Universidad de Ibagué, Carrera 22 Calle 67, Ibagué 730001, Colombia
} 


\title{
DETERMINATION OF MORIN IN GREEN TEA AND COFFEE BY ADSORPTION VOLTAMMETRY USING CARBON ELECTRODES MODIFIED WITH POLY (3,4-ETHYLENEDIOXYTHIOPHENE) AND IONIC LIQUID
}

\begin{abstract}
This paper presents a sensitive and selective method for morin (MR) determination in the presence of rutin (RT) and quercetin (QC) on a screen-printed carbon electrode coated with poly(3,4-ethylenedioxythiophene) (PEDOT) and ionic liquids 1-butyl-3-methylimidazolium tetrafluoroborate $\left(\mathrm{BMIMBF}_{4}\right),\left(\mathrm{PEDOT}-\mathrm{BMIMBF}_{4} / \mathrm{SPCE}\right)$. Several studies have shown that morin may be adsorbed onto the surface of the SPC electrode modified with PEDOT. Moreover, $\left(\mathrm{BMIMBF}_{4}\right)$ on the surface of the modified electrode with PEDOT increased the anodic peak current nearly $52,0 \%$ and facilitated the oxidation of morin to less positive potential values proving be an ideal surface toward the oxidation of morin. On the other hand, potential substances that may interfere with the signal of morin, such as rutin and quercetin, are oxidized to potential more and less positive respectively. Cyclic voltammetry (CV) and electrochemical impedance spectroscopy (EIS) were used to quantify and characterize the modified electrode, $\mathrm{pH}$ and electrochemical parameters such as potential adsorption, adsorption time and scan rate were optimized $\left(\mathrm{pH} 2,7 ; \mathrm{E}_{\mathrm{ADS}},-0,10 \mathrm{~V} ; \mathrm{tADS}, 60 \mathrm{~s}\right.$; and v, 50 $\mathrm{mVs}^{-1}$ ). The detection limit was $0,16 \mu \mathrm{mol} \mathrm{L}^{-1}$ and the RSD was 5,2\%. The usefulness of the modified electrode was used to quantify morin in green tea samples in the presence of other flavones with consistent result.
\end{abstract}

Key words: morin; green tea; adsorption voltammetry; ionic liquids; screen printed carbon electrode.

\section{INTRODUCCIÓN}

Los flavonoides que se encuentran en las plantas, principalmente frutas y verduras, contribuyen al color y al sabor. Estas sustancias son una familia de compuestos que contienen anillos aromáticos con grupos $\mathrm{OH}$ unidos los cuales son los responsables de la gran actividad antioxidante de estas sustancias. Los grupos que tienen las reacciones de transferencia de carga en los flavonoides son: pirogalol, catecol, el doble enlace en la posición 2.3 junto con el grupo 4-oxo y el grupo 3-hidroxiloyl ${ }^{1,2}$. 


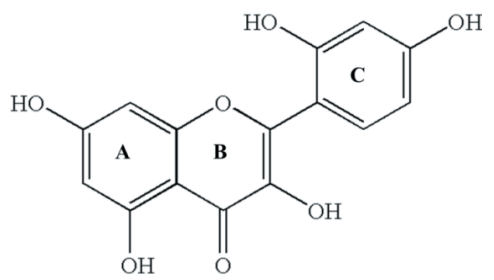

Figura 1. Estructura química de morina

La morina (3.4 ', 2', 5.7-pentahidroxiflavona) figura1, es un flavonoide activo que se encuentra en las selvas de Brasil. Se ha informado que puede proteger múltiples células en los tejidos cardiovasculares ${ }^{3}$. Sin embargo, la actividad de quercetina (2.3 ', 4', 5.7-pentahidroxiflavona) es el doble de morina ${ }^{1}$, lo que indica la importancia del 2-OH adyacente en el anillo $\mathrm{C}$ de cada flavonoide. El potencial de oxidación de los flavonoides depende de las propiedades de los sustituyentes en el anillo A y B. Estos anillos son los sitios más electroactivos a valores de $\mathrm{pH}$ medianamente ácido. Tienen una gran capacidad para complejar iones metálicos en especial $\mathrm{Pb}$ y $\mathrm{Cd}^{4}$. La importancia de esta sustancia en la dieta de las personas ha aumentado el desarrollo de sensores electroquímicos para la detección en los alimentos, de los cuales los más recientes se han resumido en la tabla 1. Estos resultados muestran una gran sensibilidad, por debajo de $0,05 \mu \mathrm{g} \mathrm{L}^{-1}$, diversidad de materiales utilizados en la modificación del electrodo de trabajo y una amplia variedad de muestras analizadas. Por otro lado, los líquidos iónicos se han utilizado cada vez más en la modificación de electrodos debido a su amplio potencial electroquímico y conductividad ${ }^{5}$. El uso de líquidos iónicos en la detección electroanalítica de morina ha sido poco, de los cuales los más utilizados son el butil-1-metil pirrolidinium (trifluorometil sulfonil) amida ${ }^{6}$ y 1-butil-3-metil-imidazolio-hexafluorofosfato ${ }^{8}$.

Tabla 1. Electrodos modificados para MR.

\begin{tabular}{c|c|l|c|c}
\hline Técnica & Electrodo de trabajo & Aplicación & $\begin{array}{c}\text { LD } \\
\left(\boldsymbol{\mu m o l ~ \mathbf { L } ^ { - 1 } )}\right.\end{array}$ & Ref. \\
\hline $\mathrm{DPV}$ & $\mathrm{V}_{2} \mathrm{O}_{5} \mathrm{NF} / \mathrm{GCE}$ & Frutas & 0,050 & 6 \\
SWV & AgNPsAETGO/GCE & Uvas & 0,003 & 8 \\
DPV & SWNT-COOH/GCE & Mora & 0,028 & 9 \\
\hline
\end{tabular}

GCE: electrodo de carbón vítreo; CPE: electrodo de pasta de carbón; MWCNT: nano tubos de carbón de pared múltiple; DPV: voltamperometría de pulso diferencial; SWV: voltamperometría de onda cuadrada; $\mathrm{CV}$ : voltamperometría cíclica; $\mathrm{V}_{2} \mathrm{O}_{5} \mathrm{NF}$ : nono hojas de óxido de vanadio; SWNT-COOH, nano tubos de carbón carboxílicos.

Este trabajo tuvo como objetivo desarrollar un nuevo método electroanalítico aplicado a la determinación de morina en té verde comercial e infusión de hierba luisa (Cymbopogon citratus) en presencia de rutina y quercetina. Por otra parte, SPCE modificado con el polímero del monómero etilendioxitiofeno (EDOT) y líquido iónico (BMIMBF4) aún no ha sido informado para la detección de morina en el té verde. 


\section{PARTE EXPERIMENTAL}

\section{Instrumentos}

Los voltamperogramas de barrido lineal y cíclicos se obtuvieron con un potenciómetro DropSens $\mu$ Stat400. La celda electroquímica estaba constituida por un sistema integrado de tres electrodos serigrafiados (DRP C110. DropSens, de Oviedo, España) con un electrodo de trabajo de carbono (4 mm), un electrodo de referencia de Ag y otro electrodo de carbono como contra electrodo. Las mediciones de $\mathrm{pH}$ se realizaron con un medidor de $\mathrm{pH} / \mathrm{mV}$ digital Orion -430 equipado con un electrodo de vidrio de $\mathrm{pH}$ combinado.

\section{Reactivos}

Se obtuvo agua de un sistema de purificación Wasselab. El etanol, el ácido nítrico y el ácido fosfórico fueron de grado analítico. Las soluciones patrón de $\mathrm{Fe}(\mathrm{CN})_{6}^{3-/ 4-}$, MR, QC y RT (Sigma-Aldrich) se prepararon una sola vez para todo el estudio $\left(5 \mathrm{mmol} \mathrm{L}^{-1}\right)$ en metanol. Se obtuvieron 3,4-etilendioxitiofeno (EDOT) y tetrafluoroborato de 1-butil-3-metilimidazolio $\left(\mathrm{BMIMBF}_{4}\right)$ de Sigma-Aldrich. Se usaron soluciones tampón de fosfato para investigar la influencia del $\mathrm{pH}$. Estos tampones se prepararon entre $\mathrm{pH} 2,0$ y 7,0 con soluciones de ácido fosfórico $0,10 \mathrm{~mol} \mathrm{~L}^{-1}$, fosfato de sodio y fosfato de di-sodio.

\section{Preparación de los electrodos modificados PEDOT/SPCE y PEDOT-BMIMBP $/$ SPCE $_{4}$}

Antes de cada preparación, el electrodo de trabajo se lavó con tampón fosfato $(\mathrm{pH} \mathrm{7,0)} \mathrm{y} \mathrm{fue}$ secado con aire caliente. El PEDOT-BMIMBP 4 /SPCE modificado se preparó en el siguiente

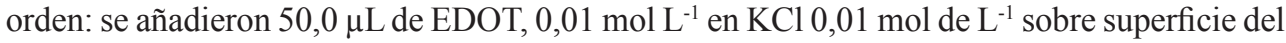
SPCE. La electro polimerización se desarrolló con 10 ciclos por voltamperometría cíclica a un voltaje de barrido entre $-0,3$ y $1,0 \mathrm{~V}$ con una velocidad de barrido de $50,0 \mathrm{mVs}^{-1}$, después de lavar PEDOT/SPCE modificado para eliminar el exceso de disolvente y se sometió a 5 ciclos de potencial entre $-0,3$ y $1.2 \mathrm{~V}\left(100 \mathrm{mV} \mathrm{s}^{-1}\right)$ para obtener una superficie estable, reproducible y limpia. Luego, se añadieron $20,0 \mu \mathrm{L}$ de $\mathrm{BMIMBP}_{4}$ sobre la reciente superficie de PEDOT/ SPCE durante 1 hora. Por último, PEDOT-BMIMBP 4 /SPCE se lavó para eliminar el exceso de disolvente. Se usó el mismo electrodo en una serie de mediciones. La preparación del electrodo modificado se muestra en la figura 2. 


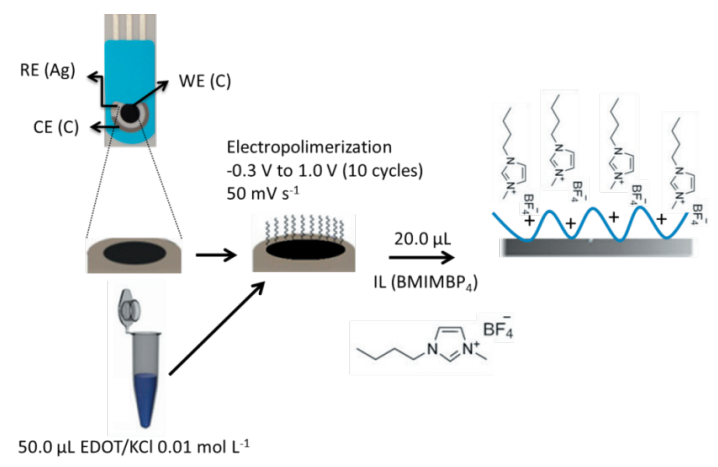

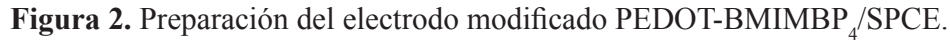

Preparación de la muestra de té verde e infusión de hierba luisa (Cymbopogon citratus). Se trataron entre 1,0 y 3,0 gramos de té verde y Cymbopogon citratus con $50 \mathrm{~mL}$ de agua caliente. La extracción se realizó durante una hora. A pesar del hecho de que las infusiones de té (negro, verde) contienen otras flavonas como RT y QC, que pueden causar interferencia en la detección de MR, las muestras no necesitan ser filtradas antes de cada medición.

\section{Procedimiento de mediciones}

En la celda electroquímica, en orden, se añadieron $7,5 \mathrm{~mL}$ de agua ultra pura, $0,5 \mathrm{~mL}$ de PBS $\left(0,01 \mathrm{~mol} \mathrm{~L}^{-1}\right)$ y entre $10-100 \mu \mathrm{L}$ de MR 5,0-0,50 $\mathrm{mmol} \mathrm{L}^{-1}$. Después de un tiempo de equilibrio de $3 \mathrm{~s}$, se registraron los voltamperogramas de barrido lineal y cíclicos (velocidad de barrido $50 \mathrm{mV} \mathrm{s}^{-1}$ ), mientras que el potencial se escaneó de 0,0 a 0,5 V. Cada voltamperograma se repitió tres veces. Las curvas de calibración se obtuvieron variando la concentración de MR entre 0,60 y 30,0 $\mu \mathrm{mol} \mathrm{L} \mathrm{L}^{-1}$. Los límites de detección se calcularon con el error estándar de la curva $(3 \sigma)$ y la pendiente de la curva de calibración. Las mediciones de impedancia electroquímica (EIS) se realizaron con el objetivo de caracterizar la respuesta electroquímica de los electrodos modificados. Las mediciones de EIS se llevaron a cabo en el potencial de circuito abierto utilizando una amplitud de perturbación de $10 \mathrm{mV}$ y un rango de frecuencia entre $10,0 \mathrm{kHz}$ y $0,10 \mathrm{~Hz}$. Los electrodos de trabajo fueron un electrodo de carbono serigrafiado (SPCE), SPCE modificado con PEDOT (PEDOT/SPCE) y SPCE modificado con PEDOT y $\mathrm{BMIMBF}_{4}\left(\mathrm{PEDOT} \mathrm{BMIMBF}_{4} / \mathrm{SPCE}\right)$ y el electrólito de prueba fue $\mathrm{Fe}(\mathrm{CN})_{6}{ }^{3-/ 4-} 10,0 \mathrm{mmol} \mathrm{L}^{-1}$ en $\mathrm{KCl} 10,0 \mathrm{mmol} \mathrm{L}^{-1}$. Para eliminar los efectos de la matriz se utilizó el método de adición estándar. Todos los datos se obtuvieron a temperatura ambiente $\left(\sim 25^{\circ} \mathrm{C}\right)$. 


\section{RESULTADOS Y DISCUSIÓN}

\section{Caracterización de PEDOT-BMIMBP4/SPCE con $\mathrm{Fe}(\mathrm{CN})_{6}^{3-/ 4-}$ por $\mathrm{CV}$ and EIS}

Se usó una disolución de $\mathrm{Fe}(\mathrm{CN}) 6^{3-/ 4-} 10,0 \mathrm{mmol} \mathrm{L}^{-1}$ en $\mathrm{KCl} 10,0 \mathrm{mmol} \mathrm{L}^{-1}$ para identificar las características generales y caracterizar la superficie por voltamperotría cíclica $(\mathrm{CV})$ e impedancia electroquímica (EIS). Los resultados se muestran en la figura 3. Usando CV, se observaron corrientes pico anódicas y catódicas para el sistema $\mathrm{Fe}^{3+}+\mathrm{e}^{-} \leftrightarrow \mathrm{Fe}^{2+}$ a 0,33 y $0,036 \mathrm{~V}$, respectivamente. Con $\triangle \mathrm{E}$ 0,29 V usando SPCE sin ser modificado (Fig. 3A curva negra). Cuando SPCE se modificó con PEDOT (PEDOT/SPCE), las corrientes pico anódicas y catódicas cambiaron a valores menos positivos de 0,26 y $0,028 \mathrm{~V}$, respectivamente. Con $\triangle \mathrm{E}$ 0,23 V (Fig. 3A curva roja). Estos resultados indican que PEDOT se ha depositado en la superficie de SPCE. Además, condujo a una disminución en la cantidad de energía para el proceso de redox. Algunos informes confirman que PEDOT facilita la transferencia de electrones en otros electrodos modificados ${ }^{10}$. Por otro lado, cuando PEDOT/SPCE se modificó con $\mathrm{BMIMBF}_{4}$, el potencial máximo anódico y catódico se observó a 0,30 y $-0,026 \mathrm{~V}$ respectivamente. Con $\Delta \mathrm{E} 0,34 \mathrm{~V}$ (Fig. 3A curva azul). Además, las corrientes pico anódicas y catódicas aumentaron 30 y $27 \%$, respectivamente. Estos resultados indican que la presencia de $\mathrm{BMIMBF}_{4}$ en la superficie de PEDOT/SPCE favoreció la transferencia de electrones para el sistema $\mathrm{Fe}^{3+}+\mathrm{e}^{-} \leftrightarrow \mathrm{Fe}^{2+}$ aunque requiere más energía. Resultados similares fueron reportados previamente usando PEDOT e IL en nanotubos de carbono de pared simple ${ }^{11}$.
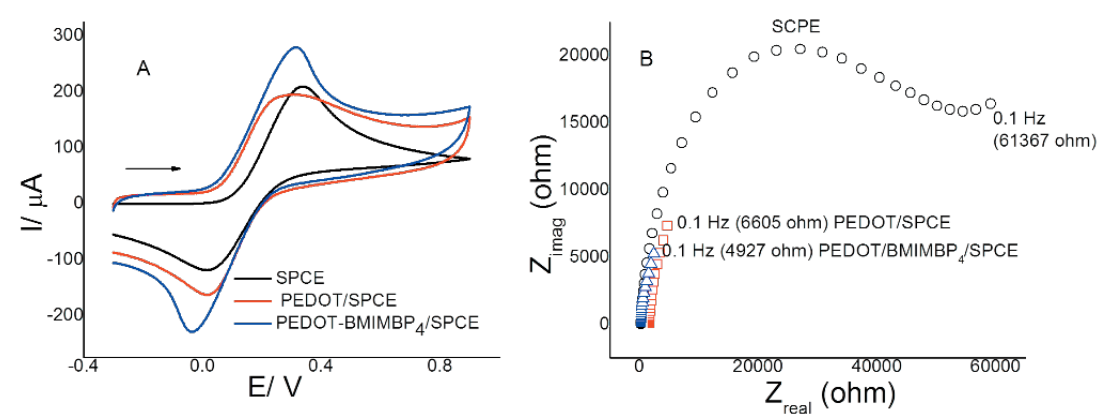

Figura 3. A) Voltamperogramas cíclicos de $\mathrm{Fe}(\mathrm{CN})_{6}^{3-44-10.0 ~} \mathrm{mmol} \mathrm{L}^{-1}$ en solución $\mathrm{KCl} 10 \mathrm{mmol} \mathrm{L}^{-1}$ usando SPCE (curva negra), PEDOT/SPCE (curva roja) y

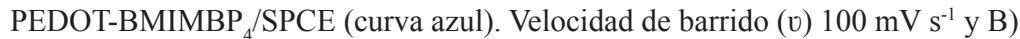
Gráfico de Nyquist para $\mathrm{Fe}(\mathrm{CN})_{6}^{3-/ 4-10,0} \mathrm{mmol} \mathrm{L}^{-1}$ en $\mathrm{KCl} 10,0 \mathrm{mmol} \mathrm{L}^{-1}$.

El análisis de impedancia electroquímica normalmente se informa en el gráfico de Nyquist, donde la resistencia real $\left(Z_{\text {real }}\right)$ se representa frente a la resistencia imaginaria $\left(Z_{\text {imag }}\right)$ para un circuito eléctrico a frecuencias altas y bajas. Los altos valores de resistencia indican un alto impedimento para la transferencia de electrones ${ }^{12}$. El gráfico de Nyquist del EIS para $\mathrm{Fe}(\mathrm{CN})_{6}^{3-/ 4-}$ usando SPCE, PEDOT/SPCE y PEDOT-BMIMBP ${ }_{4} / \mathrm{SPCE}$ se muestra en la figura 3B. La gráfica de Nyquist mostró un semicírculo usando SPCE sin modificar en la parte de alta 
frecuencia con alta resistencia a la transferencia de carga $\mathrm{R}_{\mathrm{ct}}(60 \mathrm{k} \Omega)$ que sugiere el transporte de masa por difusión. Usando PEDOT/SPCE y PEDOT-BMIMBP4/SPCE, los valores de Rct son más bajos que $\mathrm{R}_{\mathrm{ct}}$ del sustrato SPCE. Este resultado indica que la modificación de la superficie del electrodo SPCE con PEDOT y BMIMBP fue efectiva para aumentar tanto la conductividad eléctrica como la respuesta electroquímica del electrodo. Por otra parte, PEDOT aumentó el área de superficie activa como ha sido reportado previamente ${ }^{13}$.

\section{Comportamiento electroquímico de MR usando SCPE modificado con PEDOT y BMIMBP $_{4}$.}

Las propiedades electroactivas de $\mathrm{MR}$ dependen de los $\mathrm{OH}$ adyacente al anillo $\mathrm{C}$ (figura $1)^{5,14}$. La figura 4 muestra los voltamperogramas cíclicos de $\mathrm{MR}\left(0,14 \mathrm{mmol} \mathrm{L}{ }^{-1}\right)$ a $50 \mathrm{mV} \mathrm{s}^{-1}$ en pH 3.0 con PBS, usando los electrodos SPCE (curva negra), PEDOT/SPCE (curva roja), PEDOT-BMIMBP $_{4} / \mathrm{SPCE}$ (azul curva).

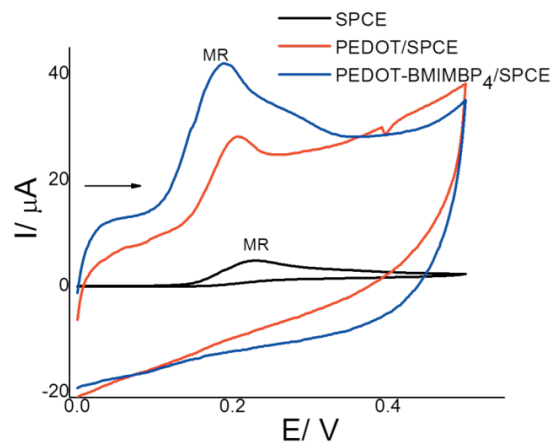

Figura 4. Voltamperogramas cíclicos de $\mathrm{MR}\left(0,14 \mathrm{mmol} \mathrm{L}^{-1}\right) \mathrm{pH} 3,0$ (PBS) usando SPCE (curva negra), PEDOT/SPCE (curva roja) y PEDOT-BMIMBP $/ 4$ SPCE (curva azul). Velocidad de barrido (v) $50,0 \mathrm{mV} \mathrm{s}^{-1}$.

Usando SPCE, se observó una intensidad de corriente anódico para MR (curva negra) a 0,22 $\mathrm{V}(4,01 \mu \mathrm{A})$. Con PEDOT/SPCE modificado (curva roja) el valor de potencial se desplazó a valores potenciales menos positivos de $0,20 \mathrm{~V}$. Además, la corriente anódica aumento $(10,7$ $\mu \mathrm{A})$. Posiblemente, este aumento de la corriente para MR se debió a un proceso de afinidad con el área hidrofóbica y no por la carga positiva del PEDOT a este valor de $\mathrm{pH}$, donde MR no ha perdido protones y, por lo tanto, no presenta carga. La interacción entre MR y PEDOT no ha sido reportada previamente para hacer una comparación más concluyente. Cuando PEDOT/SPCE se modificó con BMIMPB4, la corriente máxima anódica aumenta a 19,2 $\mu \mathrm{A}$. BIMIPB4 presentó un efecto catalítico sobre la corriente pico anódica para MR, mejorando la sensibilidad. Este electrodo modificado mostró una gran capacidad para la oxidación de la morina. Este electrodo se escogió como óptimo para los estudios posteriores 


\section{Influencia de la velocidad de barrido de MR usando PEDOT-BIMIBP $/ \mathrm{SPCE}_{4}$}

Con el objetivo de identificar el proceso de transferencia de masa, se estudió la influencia de la velocidad de barrido (v) sobre la corriente anódica para $\mathrm{MR}\left(0,14 \mathrm{mmol} \mathrm{L}^{-1}\right)$ usando PEDOT-BMIMBP $_{4} / \mathrm{SPCE}$ (figura 5). La corriente anódica se estudió entre $10-80 \mathrm{mV} \mathrm{s}^{-1}$. La ecuación de regresión fue $\mathrm{I}_{\mathrm{pa}}(\mu \mathrm{A})=3,30+0,2711 \mathrm{v}\left(\mathrm{mV} \mathrm{s}^{-1}\right)$, con coeficiente de correlación $\mathrm{r}=0,997$. Esto indica que el proceso está controlado por adsorción. A valores de velocidad más altas, la corriente máxima disminuye, lo que indica que el proceso se vuelve más lento por incremento en la saturación del electrodo. Se informaron resultados similares para MR usando nanotubos de carbono de pared simple ${ }^{7}$.
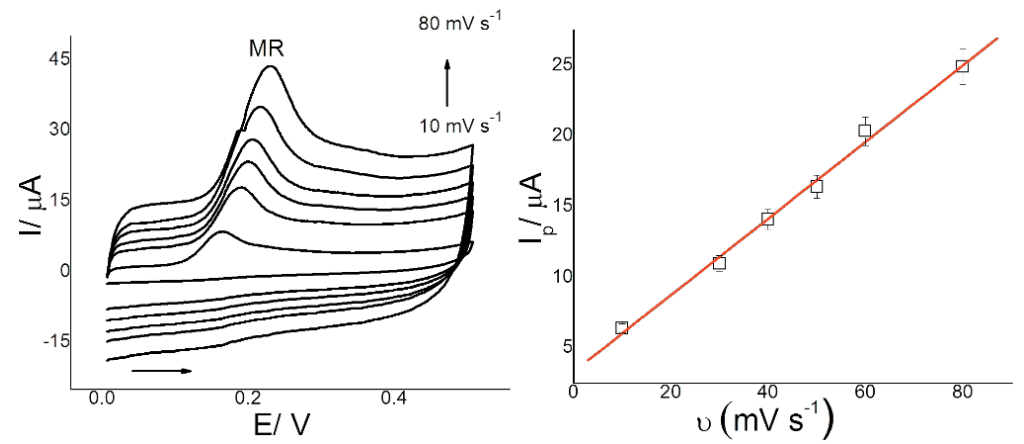

Figura 5. Voltamperogramas cíclicos y dependencia de la corriente anódica sobre la velocidad de barrido para MR $\left(0,14 \mathrm{mmol} \mathrm{L}^{-1}\right)$. $\mathrm{pH} 3,0$ (PBS) usando PEDOT$\mathrm{BIMIBP}_{4} / \mathrm{SPCE}$.

\section{Efecto del pH}

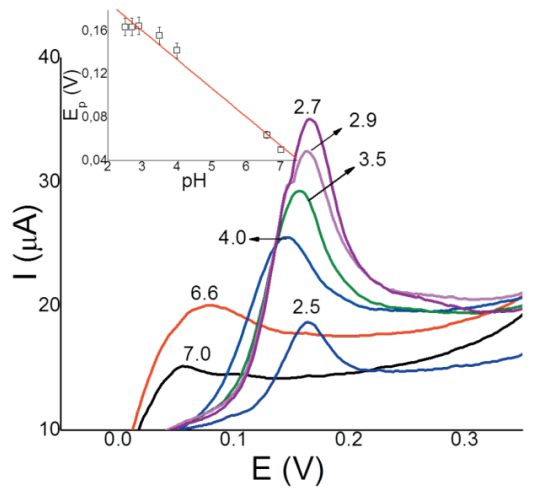

Figura 6. Voltamperogramas cíclicos del efecto de $\mathrm{pH}$ sobre la corriente anódica para MR 0,14 mmol L-1 usando PEDOT-BMIMBP4/SPCE. Condiciones: velocidad de barrido $50 \mathrm{mV} \mathrm{s}^{-1}$. 
La corriente anódica para MR $0,14 \mathrm{mmol} \mathrm{L}^{-1}$ como una función de $\mathrm{pH}$ se estudió entre valores de $\mathrm{pH}$ 2,0-7,0 con PBS (figura 6) por voltamperometría cíclica usando PEDOT-BIMIBP 4 SPCE. La corriente máxima anódica para MR fue observada a $\mathrm{pH}$ 2.7. Esto puede explicarse en función de los altos valores de $p \mathrm{~K}_{\mathrm{a}}$ para $\mathrm{MR}\left(p \mathrm{~K}_{1}=4.7 ; p \mathrm{~K}_{2}=7.8 ; p \mathrm{~K}_{3}=9.8 ; p \mathrm{~K}_{4}=\right.$ $10.7)^{4}$. A valores de $\mathrm{pH}$ inferiores de 4,0 , la $\mathrm{MR}$ no ha perdido protones $\left(\mathrm{H}^{+}\right)$. El cambio de los valores de potencial anódicos para MR hacia valores menos positivos con el aumento del $\mathrm{pH}$ (inserto en la figura 6) fue lineal entre $\mathrm{pH} 7,0$ y 2,9 con una pendiente ( $\mathrm{p}$ ) de $-0,029 \mathrm{~V}$, estos resultados sugieren que los protones $\left(\mathrm{H}^{+}\right)$están involucrados en la oxidación de $\mathrm{MR}$.

\section{Efecto del potencial y tiempo de adsorción (EADS, tADS) sobre la corriente anódica para MR.}

El efecto del potencial y tiempo de adsorción sobre la corriente anódica máxima de MR a pH 3,7 se estudió mediante voltamperometría de adsorción de barrido lineal (LSAdV). Las condiciones experimentales fueron: MR 0,60 $\mu \mathrm{mol} \mathrm{L}^{-1}$ y velocidad de exploración $50 \mathrm{mV} \mathrm{s}^{-1}$. El tiempo y el potencial de adsorción están asociados con la sensibilidad. El valor óptimo para este potencial de adsorción $\left(\mathrm{E}_{\mathrm{ADS}}\right)$ fue de $-0,1 \mathrm{~V}$. Este potencial permitió el depósito de MR por adsorción. El valor óptimo para el tiempo de adsorción $\left(\mathrm{t}_{\mathrm{ADS}}\right)$ fue de $60 \mathrm{~s}$. Para tiempos de absorción muy largos, la superficie del electrodo se saturó y la corriente anódica descendió.

\section{Límite de detección}

En orden de obtener el límite de detección de MR por LSAdV usando PEDOT-BMIMBP ${ }_{4}$ SPCE, se utilizaron las condiciones optimizadas: pH 2,7 (0,01 $\left.\mathrm{mol} \mathrm{L}^{-1} \mathrm{PBS}\right)$, EADS, -0,10 $\mathrm{V}$; tADS, 60s; y velocidad de barrido $50 \mathrm{mVs}^{-1}$. La figura 7 muestra los voltamperogramas

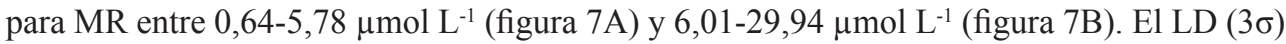
obtenido fue de $0,16 \mu \mathrm{mol} \mathrm{L^{-1 }}$ y la desviación estándar relativa fue de 5,5\% con MR 6,0 $\mu \mathrm{mol} \mathrm{L}-1(\mathrm{n}=5)$
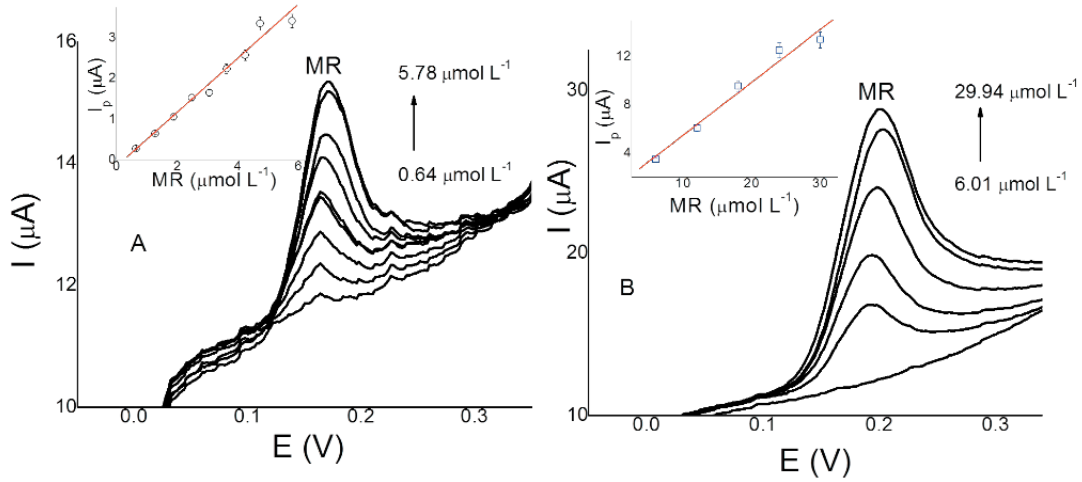

Figura 7. voltamperogramas de barrido lineal para MR entre $0,64-5,78 \mu \mathrm{mol} \mathrm{L} \mathrm{L}^{-1}$ (A) y 6,01-29,94 $\mu \mathrm{mol} \mathrm{L}^{-1}$ (B) usando PEDOT-BMIMBP4/SPCE. Condiciones: $\mathrm{pH} 2,7 ; \mathrm{E}_{\mathrm{ADS}}-0,10 \mathrm{~V} ; \mathrm{t}_{\mathrm{ADS}} 60 \mathrm{~s}$ y velocidad de barrido $50 \mathrm{mV} \mathrm{s}^{-1}$. En cada figura se muestra la curva de calibrado. 


\section{Validación y estudio de interferencias}

El efecto de posibles sustancias interferentes en la detección de MR usando PEDOT$\mathrm{BMIMBP}_{4} / \mathrm{SPCE}$ se evaluó en presencia de otros flavonoides como RT y QC. La figura 8 muestra las corrientes pico anódicas para QC, MR 6,0 $\mu \mathrm{mol} \mathrm{L} \mathrm{L}^{-1}$ y RT 60,0 $\mu \mathrm{mol} \mathrm{L} \mathrm{L}^{-1}$. Los resultados muestran un potencial de separación para MR-QC de $50 \mathrm{mV}$. Además, la corriente anódica fue mayor para MR. El potencial de separación para MR-RT fue de $380 \mathrm{mV}$. Por lo tanto, PEDOT-BMIMBP 4 /SPCE presentó gran selectividad para MR.

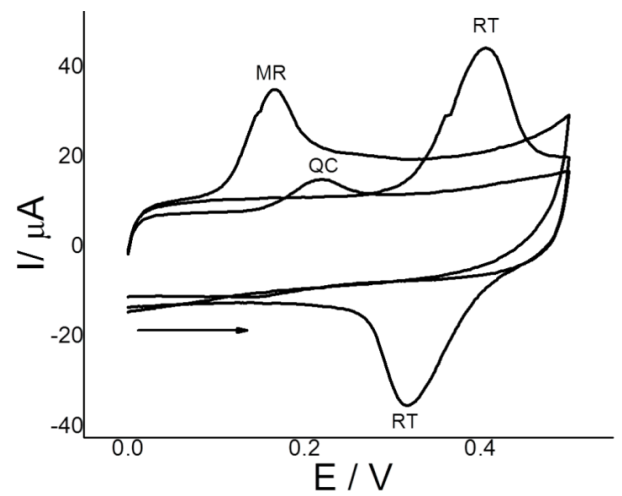

Figura 8. Voltamperogramas de barrido lineal para MR, QC $6.0 \mu \mathrm{mol} \mathrm{L}^{-1}$ y RT 60,0 $\mu \mathrm{mol} \mathrm{L}^{-1}$, usando PEDOT-BMIMBP ${ }_{4} /$ SPCE$_{\text {. Condiciones: } \mathrm{pH}}$ 2,7; EADS -0,10 V; tADS $60 \mathrm{~s}$ y velocidad de barrido $50 \mathrm{mV} \mathrm{s}^{-1}$

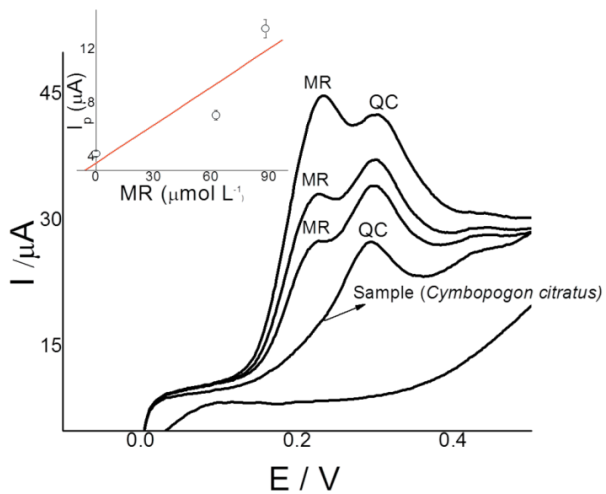

Figura 9. Voltamperogramas de barrido lineal y curva de calibrado para la muestra $1 *$ (tabla 2), usando PEDOT-BMIMBP4/SPCE. Condiciones: $\mathrm{pH} 2,7 ; \mathrm{E}_{\mathrm{ADS}}-0,10 \mathrm{~V}$; $\mathrm{t}_{\mathrm{ADS}} 60 \mathrm{~s}$ y velocidad de barrido $50 \mathrm{mV} \mathrm{s}^{-1}$. 
La utilidad del método fue validada usando infusión de hierbas de Cymbopogon citratus. Los valores obtenidos se resumen en la tabla 2. La figura 9 muestra los voltamperogramas para la muestra $1^{*}$ (tabla 2). Se observó que la matriz de la muestra no afectó la señal para MR, pero disminuyó el potencial de separación para MR-QC. Además, otras flavonoides, como RT, no mostraron señal a concentraciones 200 veces más altas. No se detectó MR, debido posiblemente a que la concentración de MR en Cymbopogon citratus puede estar por debajo del límite de detección, pero la muestra fue dopada con cantidades conocidas de MR para verificar la exactitud del método. Por otro lado, solo se detectó quercetina entre 22,0 y 37,41 $\mu \mathrm{mol} \mathrm{L} \mathrm{L}^{-1}$ en esta infusión de hierbas. Este valor detectado fue similar al reportado para frutos cítricos $^{19}$.

Tabla 2. Resultados de la validación del método para MR

\begin{tabular}{c|c|c|c|c}
\hline Muestra & $\begin{array}{c}\text { Concentración } \\
\text { inicial en muestra } \\
\text { MR }(\mu \mathrm{mol} \mathrm{L}-1)\end{array}$ & $\begin{array}{c}\text { Concentración } \\
\text { agregada a la muestra } \\
\text { MR }(\mu \mathrm{mol} \mathrm{L})^{-1}\end{array}$ & $\begin{array}{c}\text { Concentración inicial + } \\
\text { agregada } \\
\text { MR }(\mu \mathrm{mol} \mathrm{L})^{1}\end{array}$ & $\begin{array}{c}\% \text { Error } \\
\text { relativo }\end{array}$ \\
\hline $1^{*}$ & --- & 34,01 & 37,65 & 10,7 \\
2 & --- & 161,2 & 165,17 & 2,45 \\
\hline \multicolumn{4}{r}{ * Voltamperogramas y curva de calibrado son mostradas en la figura 9}
\end{tabular}

\section{Analítica aplicación}

PEDOT-BMIMBP4/SPCE se utilizó en la detección y cuantificación de MR en muestras comerciales de té verde y café obtenidas en supermercados de la ciudad de Ibagué, Colombia. Los valores de concentración de MR se calcularon por adición estándar en presencia de soluciones estándar de MR. La extracción de MR se desarrolló con agua caliente. Los resultados se muestran en la tabla 3. Los valores obtenidos para MR fueron mayores en comparación con los detectados en mora $^{9}$ y fresa ${ }^{7}$.

Tabla 3. Resultados de MR en muestras comerciales de té verde y café

\begin{tabular}{c|c|c}
\hline Muestras & Concentración de MR detectado $\left(\boldsymbol{\mu m o l ~ \mathbf { L } ^ { - 1 } \text { ) }}\right.$ & $\mathbf{\% R S D}$ \\
\hline Té $^{1}$ & 53,02 & 2,0 \\
Té $^{2}$ & 55,6 & 1,2 \\
Café & 13,0 & 0,5 \\
\hline
\end{tabular}

\section{CONCLUSIONES}

La modificación de un EPCE con PEDOT y el líquido iónico $\mathrm{BMIMBP}_{4}$ se caracterizó bien con $\mathrm{Fe}(\mathrm{CN})_{6}^{3-/ 4-}$ y MR por $\mathrm{CV}$ y EIS. El electrodo modificado se desarrolló fácilmente y fue muy conveniente para la determinación de MR en té verde. Además, otros flavonoides como RT y QC no causan interferencia. El límite de detección alcanzado fue muy cercano a previos reportes que están por debajo de $1,0 \mu \mathrm{mol} \mathrm{L}^{-1}$. Por último, la actividad del sensor en muestras reales con una matriz más compleja no fue afectada. 


\section{AGRADECIMIENTOS}

Universidad de Ibagué por el financiamiento de los proyectos 15-376-INT y 16-416-INT.

\section{REFERENCIAS BIBLIOGRÁFICAS}

1. Heijnen CGM, Haenen GRMM, Vekemans JAJM, Bast A. Peroxynitrite scavenging of flavonoids: structure activity relationship. Environ. Toxicol.Pharmacol 2001; 10: 199206.

2. Yang B, Kotani A, Arai Kusu KF. Estimation of the antioxidant activities of flavonoids from their oxidation potentials. Anal Sci. 2001; 17: 599-604.

3. Liu W, Guo R. The interaction between morin and CTAB aggregates. J Colloid Interface Sci. 2005; 290: 564-573.

4. Kopacz M. Quercetin and Morin sulfonates as Analytical Reagents. J Anal Chem. 2003; 58: 225-229.

5. Xiao Y, Zhang H. Homogeneous ionic liquid microextraction of the active constituents from fruits of Schisandra chinensis and Schisandras phenanthera. Anal Chim Acta. 2012; 712: 78-84.

6. Sasikumar R, Govindasamy M, Chen SM, Chieh-Liu Y, Ranganathan P, Rwei SPJ. Electrochemical determination of morin in Kiwi and Strawberry fruit samples using vanadium pentoxide nano-flakes. J Colloid Interface Sci. 2017; 504: 626-632.

7. Mariño, A, Leiva Y, Bolaños K, García-Beltrán O, Nagles E, Determination of pentahydroxyflavones using coated chitosan multi-wall carbon nanotubes and an ionic liquid glassy carbon electrode by adsorption stripping voltammetry (AdSV). J Electroanal Chem. 2015; 759: 153-157.

8. Yola M, Gupta V, Eren T, Sen A, Atar N. A novel electro analytical nanosensor based on graphene oxide/silver nanoparticles for simultaneous determination of quercetin and morin. Electrochim Acta. 2014; 120: 204-211.

9. Ziyatdinova G, Ziganshina E, Budniko H. Electrooxidation of morin on glassy carbon electrode modified by carboxylated single-walled carbon nanotubes and surfactants. Electrochim Acta. 2014; 145: 209-216.

10. Zhang K, Xu J, Zhu X, Lu L, Duan X, Hu D, et. al. Poly(3,4-ethylenedioxythiophene) nanorods grown on graphene oxide sheets as electrochemical sensing platform for rutin. J Electroanal Chem. 2015;739: 66-72.

11 Nagles E, García-Beltrán O. Determination of Rutin in Black Tea by Adsorption Voltammetry (AdV) in the Presence of Morin and Quercetin. Food Anal Methods. 2016; 9: 3420-3427.

12. Ensafi A, Arashpour B, Rezaei B, Allafchian A. Voltammetric behavior of dopamine at a glassy carbon electrode modified with $\mathrm{NiFe} 2 \mathrm{O} 4$ magnetic nanoparticles decorated with multiwall carbon nanotubes. Mat Sci Eng C. 2014; 39: 78-85. 
13. Serafín V, Agüí L, Yáñez-Sedeño P, Pingarrón JM. A novel hybrid platform for the preparation of disposable enzyme biosensors based on poly (3,4-ethylenedioxythiophene) electrodeposition in an ionic liquid medium onto gold nanoparticles-modified screenprinted electrodes. J Electroanal Chem. 2011; 656: 152-158.

14 Liu W, Guo R. Interaction between flavonoid, quercetin and surfactant aggregates with different charges. J Colloid Interface Sci. 2006; 302: 625-632.

15. Godwin A, Daniel D, Shadrack SA, Elom KA, Nana Afua B, Godsway KG, et. al. Determination of elemental, phenolic, antioxidant and flavonoid properties of Lemon grass (CymbopogoncitratusStapf). Int Food Research J. 2014; 21: 1971-1979. 